\title{
Discovery of Process-Induced Tetragonality in Equiatomic Ferromagnetic FeNi
}

\author{
A. M. Montes-Arango ${ }^{\mathrm{a}}$, L. G. Marshall ${ }^{\mathrm{b}, \mathrm{c}}$, A. D. Fortes ${ }^{\mathrm{d}, \mathrm{e}, \mathrm{f}}$, N. C. Bordeaux ${ }^{\mathrm{b}}$, S. Langridge $\mathrm{f}^{\mathrm{f}}$ \\ K. Barmak ${ }^{\mathrm{g}}$, and L. H. Lewis ${ }^{\mathrm{a}, \mathrm{b}, \mathrm{c}}$
}

${ }^{a}$ Department of Mechanical and Industrial Engineering, Northeastern University, 360 Huntington Avenue, Boston, Massachusetts 02115, USA;

${ }^{\mathrm{b}}$ Department of Chemical Engineering, Northeastern University, 360 Huntington Avenue, Boston, Massachusetts 02115 USA;

${ }^{c}$ The George J. Kostas Research Institute for Homeland Security at Northeastern University, LLC, 141 S Bedford St, Burlington, MA USA;

${ }^{\mathrm{d}}$ Department of Earth and Planetary Sciences, Birkbeck, University of London, Malet Street, London WC1E 7HX, United Kingdom;

${ }^{\mathrm{e}}$ Department of Earth Sciences, University College London, Gower Street, London WC1E 6BT, United Kingdom;

${ }_{\mathrm{f}}^{\mathrm{f}}$ ISIS Facility, Rutherford Appleton Laboratory, Harwell Science and Innovation Campus, Didcot, Oxfordshire OX11 0QX United Kingdom;

${ }^{g}$ Department of Applied Physics and Applied Mathematics, Columbia University, New York, New York 10027, USA.

Corresponding author: Prof. Laura H. Lewis, 313 Snell Engineering Center, Northeastern University, 360 Huntington Ave, Boston, Massachusetts 02115, USA. Phone: +1-617-373-3419; Email:1hlewis@neu.edu 
Abstract: Synthesis of a new tetragonal phase at the equiatomic composition in the archetypal binary Fe-Ni phase diagram is reported. This new phase is proposed as a transitional phase linking cubic FeNi with the chemically ordered tetragonal $\mathrm{L}_{0} \mathrm{FeNi}$ compound, tetrataenite, of interest as a new advanced permanent magnetic material. This new tetragonal phase was created in a selection of nominally equiatomic FeNi alloys, made either from natural $\mathrm{Fe}$ and $\mathrm{Ni}$ or from natural Fe combined with the ${ }^{62} \mathrm{Ni}$ isotope, via application of high-strain processing methods followed by an annealing protocol. High-resolution neutron diffraction affirms that all unprocessed samples adopt the A1-type cubic structure (space-group $F m \overline{3} m$ ) while all fully processed samples adopt the chemically disordered A6-type tetragonal structure (space-group $14 / \mathrm{mmm}$ ). Magnetic characterization documents a decrease in the initial magnetic susceptibility of deformed samples after annealing, evidencing a processing-induced increase in magnetic anisotropy that may be entirely accounted for by the measured tetragonal distortion. It is proposed that this new phase is a precursor to the formation of tetrataenite $\left(\mathrm{L}_{0} \mathrm{FeNi}\right.$, space-group $\left.\mathrm{P} 4 / \mathrm{mmm}\right)$, a meteoritic mineral of high magnetization and appreciable magnetocrystalline anisotropy that requires extraordinarily long cooling periods to form in nature. These results furnish new fundamental information as well as engineering insight for terrestrial synthesis of tetrataenite on industrial timescales, with high relevance for the creation of next-generation permanent magnets comprised entirely of easily accessible, earth-abundant elements.

Keywords: tetragonal distortion; order-disorder phase transformations; plastic deformation; neutron diffraction; $\mathrm{L} 1_{0} \mathrm{FeNi}$; permanent magnets; 


\section{Introduction}

Alloys comprised of iron and nickel have been essential to human civilization, with evidence of artifacts fashioned from meteoritic FeNi dated to the $5^{\text {th }}$ millennium BCE [1,2], and from smelted FeNi alloys dated as early as the $3^{\text {rd }}$ millennium BCE [3]. Further, modern metallurgy has been built, in large part, on these alloys, which all adopt cubic crystal structures. However, a lowersymmetry tetragonal phase of $\mathrm{FeNi}$, known as tetrataenite, has been identified by the planetary science community as a component of many stony, stony-iron, and iron meteorites and has rekindled interest in this historic alloy system. The mineral tetrataenite features a high magnetization and a room-temperature magnetocrystalline anisotropy energy density in the range 1.0-1.3 MJ $/ \mathrm{m}^{3}$ [4]. This appreciable anisotropy energy value, attributed to tetrataenite's chemically ordered tetragonal crystal structure (space group $P 4 / m m m, ~ L 1_{0}$ Strukturbericht designation), advances $\mathrm{L}_{1}$-type $\mathrm{FeNi}$ as a promising permanent magnet candidate with a technologically relevant theoretical maximum energy product of $335 \mathrm{~kJ} / \mathrm{m}^{3}$ (42 MGOe) [4], approximately $66 \%$ of that of $\mathrm{NdFeB}$-based supermagnets. As indispensable technologic materials, permanent magnets permit interconversion of mechanical and electrical energy to enable critical technologies in alternative energy, communications systems and military applications, among others. In support of the global energy challenge [5,6], special attention has been devoted to the development of new magnetic materials, such as $\mathrm{L}_{1}$-type $\mathrm{FeNi}$, with the potential to supplement and/or replace rare-earth-based permanent magnets for energy transformation and generation. However, enthusiasm for incorporation of $\mathrm{L}_{0} \mathrm{FeNi}$-based magnets in technological applications is considerably tempered by its formidable synthesis requirements: natural formation of tetrataenite in meteorites requires extraordinarily long cooling periods of up to one billion years [7]. Engineering the development of tetragonality and long-range chemical ordering in the 
chemically disordered face-centered cubic (fcc or A1, space group $F m \overline{3} m$ ) parent phase to form tetrataenite has proven to be very challenging [8].

It is within this context that we describe here the synthesis of a new FeNi phase with a chemically disordered tetragonal crystal structure that may be considered as a precursor to the elusive tetrataenite phase. This new phase has been realized in a selection of FeNi-based alloys subjected to severe plastic deformation and post-deformation treatments that allow access to kinetically hindered but thermodynamically accessible states. The existence of this new phase in specifically processed FeNi-based samples is confirmed through analyses of high-resolution neutron diffraction data that unambiguously demonstrate the presence of tetragonality that is unaccompanied by detectable chemical order, describing the A6-type structure (space-group $I 4 / \mathrm{mmm})$. The absence of chemical order is confirmed through exploitation of the isotopic dependence of neutron scattering amplitudes in the materials of this study consisting of natural $\mathrm{Fe}$ alloyed with the isotope ${ }^{62} \mathrm{Ni}$. It is postulated that this chemically disordered tetragonal A6-type structure constitutes a transitional phase linking the ordinary disordered cubic A1 structure with the chemically ordered tetragonal $\mathrm{L} 1_{0}$-type compound, tetrataenite. Overall, these results provide enabling insight into the conditions and processes necessary to favor the formation of tetrataenite, as well as inform the microstructural aspects of this material in its potential transition from a research material to a commercial magnet.

The impressive permanent magnetic properties of tetrataenite are derived from, in part, its lowersymmetry tetragonal $\mathrm{L}_{0}$-type crystal structure that may be described as a low-temperature crystallographic derivative of a face-centered cubic lattice. This anisotropy contributes to a large magnetic remanence which significantly amplifies the amount of magnetic energy that may be stored in a magnet. The $\mathrm{L} 1_{0}$ crystal structure consists of alternating layers of constituent elements 
stacked along the tetragonal $c$-axis, creating a natural superlattice, Fig. 1. The $c$-axis also defines the uniaxial easy direction of magnetization and of the accompanying uniaxial magnetocrystalline anisotropy. The $\mathrm{L} 1_{0}$ phase is believed to form by nucleation and growth processes through a thermodynamically first-order chemical disorder-order phase transformation from a parent lowanisotropy fcc (A1-type) phase below a critical chemical order-disorder temperature $T_{O D}[9,10]$. While isothermal annealing at temperatures below $T_{O D}$ is usually sufficient to promote $\mathrm{L} 1_{0}$ phase formation in related systems such as FePd and FePt [11], the task of obtaining the $\mathrm{L} 1_{0}$ phase in the FeNi system is quite difficult due, in part, to its very low $T_{O D}$ of $320^{\circ} \mathrm{C}[12]$ and to the extremely sluggish diffusion rate of $\mathrm{Ni}$ in the iron host at that temperature $[8,13]$. For comparative purposes, $T_{O D}$ for the $\mathrm{L} 1_{0}$-type ferrous compounds FePd and FePt are $680^{\circ} \mathrm{C}$ and $1300{ }^{\circ} \mathrm{C}$, respectively [14], temperatures at which atomic mobilities are much greater.

Twentieth-century scientific literature contains hints of the presence of unexpected bonding in the Fe-Ni binary system. The existence of a low-temperature superlattice in the Fe-Ni system in the vicinity of the equiatomic composition was first suggested by Dahl in 1936 [15]. Dahl reported exceptional changes in the resistivity of FeNi alloys, including equiatomic FeNi, subjected to different processing conditions that was reminiscent of similar behavior exhibited by phases in the $\mathrm{Au}-\mathrm{Cu}$ system which was confirmed to contain low-temperature superstructured compounds. The presence of preferential interatomic interactions in FeNi suggesting the existence of a lowersymmetry structure was also reported by Wakelin and Yates in 1953 [16] and later by Jiang and coworkers [17] in laboratory-synthesized alloys. Petersen confirmed the existence of a metallic phase with a superstructure in an iron meteorite in 1977 [18], with the first positive identification of tetrataenite in meteoritic material attributed to Clarke and Scott in 1980 [19]. However, laboratory synthesis of the $\mathrm{L} 1_{0} \mathrm{FeNi}$ phase has only been substantiated in materials subjected to 
non-scalable processing techniques as neutron and electron radiation $[20,21,22,23]$ or synthesized by monatomic layer-by-layer deposition processes to produce ultra-thin films [24,25]. Ordered phase formation in irradiated samples was attributed to enhancement in the rate of phase transformation fostered by a high density of extrinsic lattice defects.

The $\mathrm{L} 1_{0}$-type FeNi phase is very challenging to detect in scattering experiments due to the extremely small difference of both neutron scattering lengths and X-ray scattering factors between natural Fe and natural Ni. This small difference results in Bragg superstructure peaks - which signal the presence of chemical order in the $\mathrm{L} 1_{0}$ structure - of exceedingly low intensities. Indeed, a sample of single-phase, untextured $\mathrm{L} 1_{0} \mathrm{FeNi}$ with perfect chemical order would provide an intensity of the strongest superstructure peak (001) that is approximately $0.3 \%$ of that of the most intense fundamental (111) Bragg reflection (as seen in Fig. S1). While there have been reports of $\mathrm{L} 1_{0}$ superlattice peak detection in X-ray diffraction experiments for bulk FeNi processed samples [26,27], it remains an open question as to whether the detected peaks are signatures of $\mathrm{L1}_{0}$-type chemical order or if they correspond to other phases with Bragg reflections overlapping the $\mathrm{L} 1_{0}$ FeNi superstructure peaks of interest. For example, easily produced oxides such as $\mathrm{Fe}_{2} \mathrm{O}_{3}, \mathrm{Fe}_{3} \mathrm{O}_{4}$ and $\mathrm{NiFe}_{2} \mathrm{O}_{4}$ are confirmed to exhibit a high-intensity Bragg reflection at a $d$-spacing that coincides with the (110) $\mathrm{L} 1_{0} \mathrm{FeNi}$ superlattice peak. To meet the challenge of assessing the existence of chemical order in these materials, in this work the isotopic element ${ }^{62} \mathrm{Ni}$ has been used in place of naturally occurring Ni to manipulate the geometric structure factor for Bragg neutron diffraction reflections. Natural $\mathrm{Ni}$ has a positive neutron scattering length while that of ${ }^{62} \mathrm{Ni}$ is large and negative; therefore employing the scattering lengths for natural $\mathrm{Fe}$ and for ${ }^{62} \mathrm{Ni}$ yields geometric structure factors that are very sensitive to any long-range chemical order that might be present in the $\mathrm{Fe}^{62} \mathrm{Ni}$ alloy. In detail, the coherent neutron scattering lengths of ${ }^{62} \mathrm{Ni}$ and 
of natural Fe [28] interact to produce a main $\mathrm{L}_{0}(001)$ superlattice Bragg peak intensity that is approximately 890 times larger than that of the main fundamental Bragg peak for pure, untextured, perfectly chemically ordered $\mathrm{L} 1_{0}$-type $\mathrm{Fe}^{62} \mathrm{Ni}$ (Fig. S2). This strategy of creating and probing alloys containing ${ }^{62} \mathrm{Ni}$ allows unequivocal determination of the presence of $\mathrm{L} 1_{0}$ chemical order, even for samples with small $\mathrm{L} 1_{0}$ phase volume fraction or with imperfect long-range chemical order.

The challenge to drastically increase the kinetics of $\mathrm{L}_{0}$ phase formation in the FeNi system has been approached here through the application of severe plastic deformation delivered to a selection of FeNi materials by two different methods: (i) mechanical milling at cryogenic temperatures (hereafter "cryomilling") and (ii) cold rolling, with both methods followed by postdeformation annealing protocols. These two well-known deformation techniques can produce large concentrations of lattice defects at concentrations comparable to those found near the materials' melting temperatures [29], delivering a positive effect on atomic diffusion in a variety of metallic systems, including aluminum and steel [30,31,32]. Further, milling at cryogenic temperatures avoids the occurrence of thermally driven recovery and recrystallization processes in highly deformed structures. Improvement of the low thermodynamic driving force for $\mathrm{L}_{0}$ phase formation in the FeNi system, considered to be the ultimate limiting factor for $\mathrm{L} 1_{0}$ phase attainment [33], was approached here through small additions of titanium. This alloy modification was selected, in part, on the basis of appreciable negative heats of formation of titanium-based $\mathrm{L} 1_{0}$ compounds that contain $\mathrm{Ni}[34]$.

\section{Materials and methods}

FeNi-based samples were synthesized from natural and isotopically enriched elements and processed for study, with precursor compositions and chemical homogeneity confirmed by energy 
dispersive X-ray spectroscopy (EDS) coupled to a field-emission scanning electron microscope (SEM, Hitachi S4800) or by X-ray fluorescence (Bruker M4 Tornado). Samples were cryomilled for a cumulative time of 9 hours in a surfactant mixture of oleic acid ( $25 \mathrm{wt} \%)$ in heptane (25 wt\%) within a liquid nitrogen bath (SPEX SamplePrep 6770 Freezer/Mill). The cryomilled powders were then collected and repeatedly rinsed with heptane and acetone in order to remove the surfactants. Cold rolling was carried out on samples prepared by drop-casting (samples containing natural $\mathrm{Ni}$ ) or by arc-melting (samples containing ${ }^{62} \mathrm{Ni}$ ). As-solidified alloys were homogenized at $500{ }^{\circ} \mathrm{C}$ for $100 \mathrm{~h}$ and were then cold-rolled in several steps with an applied load in the range 0.1-63.7 Tons (Stanat Rolling Mill). The percentage of cold-work, defined in terms of the initial $\left(t_{0}\right)$ and final $\left(t_{f}\right)$ thickness as $C W \%=\left(t_{0}-t_{f}\right) / t_{0}$, was in the range $83 \%-94 \%$. The deformed samples (cryomilled and cold-rolled) were then vacuum annealed at $290 \pm 5{ }^{\circ} \mathrm{C}$ for 24 30 days to activate the phase transformation. Room-temperature structural examination of the FeNi materials was conducted with both laboratory-based X-ray diffraction (XRD, PANalytical X'Pert Pro) and with time-of-flight neutron powder diffraction using the High Resolution Powder Diffractometer, HRPD, at the ISIS spallation neutron source [35]. HRPD is the highest resolution neutron diffractometer of its type in the world and provides the capability to detect the very small tetragonal distortion characteristic of tetrataenite $(c / a \approx 1.0036,[12])$. The neutron scattering data were exported in a format suitable for profile refinement $[36,37]$ and were refined using both the Rietveld and Le Bail methods to examine both the $\mathrm{L} 1_{0}$ and the $\mathrm{A} 1$ (fcc) structural models for all samples. Additionally, the data were analyzed to provide estimates of the crystallite size $(D)$ and strain ( ) through a modified Williamson-Hall approach [38]. Magnetic characterization at $T=10 \mathrm{~K}$ of square-shaped $\mathrm{FeNi}(\mathrm{Ti})$ cold-rolled samples was performed before and after annealing using a MPMS SQUID magnetometer (Quantum Design). Anisotropy constants were estimated 
from the Law of Approach to Ferromagnetic Saturation [39] applied to the initial magnetization $(M)$ data in the region $M \geq 0.95 M_{S}$, assuming a uniaxial system and using the high-field susceptibility $\left(40 \mathrm{kOe}, 4.2 \mathrm{~K}\right.$ ) reported for $\mathrm{Fe}_{50} \mathrm{Ni}_{50}$ [40]. To enable comparisons between measured and anticipated magnetic anisotropy in this system, the uniaxial strain anisotropy $K_{\sigma}$ originating from tetragonality of the FeNi lattice is calculated as $K_{\sigma}=1.5 \lambda_{s} \varepsilon E$, where $\lambda_{s}$ is the saturation magnetostriction of $\mathrm{Fe}_{50} \mathrm{Ni}_{50}$ in the [100] direction [41], $\varepsilon$ is the measured tetragonal distortion or strain, and $E$ is the elastic modulus in the [001] direction as calculated from the reported $\mathrm{Fe}_{50} \mathrm{Ni}_{50}$ elastic constants [42].

\section{Results and Discussion}

The assessed compositions (atomic\%), the isotopic status and processing protocol, and the sample designations are provided for each FeNi-based sample in Table 1. For comparative purposes, two forms of FeNi material with the approximate assessed composition $\mathrm{Fe}_{57} \mathrm{Ni}_{43}$ were also characterized: a commercial FeNi powder and a portion of the NWA 6259 meteorite confirmed to consist of the $\mathrm{L}_{0}$ phase tetrataenite [43]. The NWA 6259 meteorite has an overall Fe-rich composition of $\mathrm{Fe}_{59} \mathrm{Ni}_{41}$ and possesses a complex microstructure comprised of trivariant, polyordered domains of the chemically ordered $\mathrm{L} 1_{0}$ phase that formed from a highly textured, potentially single-crystal form of the disordered parent fcc phase [4].

Initial structural characterization employing the lower-resolution XRD probe indicated that, within equipment resolution, all precursor as-made alloys as well as all processed samples exhibited the face-centered cubic A1 structure with lattice $a$-parameters in the range $3.586 \AA-3.627 \AA$, in broad agreement with the reported range of FeNi alloy $a$-parameters and reflective of the compositional variations. Determined dimensions of the coherently scattering domains, or crystallite sizes, after deformation processing but prior to annealing were typically under $100 \mathrm{~nm}$. 
Deformation produced significant Bragg diffraction peak broadening associated with microstrain levels calculated to be in the range $0.18 \%-0.90 \%$. Further, XRD data (not shown) obtained from cold-rolled unannealed FeNi and FeNi(Ti) samples contained coexisting "doubled" Bragg peaks each sharp Bragg peak is partnered with a broad Bragg peak - consistent with the existence of surface-localized deformation producing two phases of differing lattice parameters, crystallite sizes and microstrain levels. This multiphase nature of the cold-rolled samples disappeared after annealing, while the crystallite sizes remained relatively constant for post-annealed samples subjected to both types of deformation processing. These data are available in Table S1.

Probing the samples with time-of-flight neutron HRPD provided a more nuanced and highly interesting story. This high-resolution probe revealed, within instrumental sensitivity, that all samples in the unprocessed state exhibited diffracted Bragg reflections consistent with the cubic A1 structure while all fully processed samples (deformed and annealed) were determined to adopt a tetragonal structure. A minor secondary phase of $\mathrm{FeO}$ was detected, at an abundance $<<1$ wt. $\%$. Summaries of these data are available in the Supporting Information section (Tables S1-S4). Importantly, as described later, it is confirmed that the onset of tetragonality does not occur until after annealing. Lattice parameters derived from neutron diffraction data corroborate those derived from XRD data but are accompanied by a much higher degree of precision, which allows for the distinction between the $a$ and $c$ lattice parameters in the fully processed samples to confirm tetragonality.

The above conclusions were attained by refining the diffraction data of each sample using both an cubic (fcc) and a tetragonal structural model. Two different structural refinement techniques were utilized: the Rietveld Method and the Le Bail Method. Results returned from both of these methods are in agreement, with a high goodness-of-fit; henceforth data derived from the Rietveld 
model was employed for analyses by virtue of its greater accuracy. The unprocessed samples made from natural metal sources are fit equally well by the fcc and tetragonal models, as evident by their similar goodness-of-fit parameters, Fig. 2; however, the degree of tetragonality allowed by the tetragonal model is so small that it approximates a cubic structure, with an upper bound of $0.08 \%$ (Table S2), as quantified by a calculated $c / a$ ratio of $1.00076(1)$. By contrast, the structural refinement results from the fully processed samples made from natural metal sources provided significantly improved goodness-of-fit parameters for the tetragonal model. Furthermore, as seen in Table S3, all processed samples possess a c/a ratio of at least 1.00241(6), which represents a degree of tetragonality with a lower bound of $0.24 \%$, more than three times larger than the maximum value determined for unprocessed samples. These quantitative findings can be visualized by examination of selective Bragg peak broadening that was obtained for deformed and annealed samples, Fig. 2(b,c). These broad peaks actually encompass two split peaks that originate from the tetragonal distortion; they were consistently observed across the entire diffraction pattern and are captured quantitatively by the refinement analysis. Thus, the cubic structure is confirmed as most appropriate for the unprocessed samples while the tetragonal structure is most appropriate for the fully processed samples. The lower limit on calculated differences in $c / a$ ratios between the processed and unprocessed samples is $0.00165(6)$; this value is approximately 9 times larger than the $3 \sigma$ uncertainty on the difference. While small, this difference is highly statistically significant. The degrees of tetragonality, as quantified by the calculated $c / a$ ratios, were determined for the fully processed (deformed, post-annealed) samples of cryomilled FeNi(Ti), cold-rolled FeNi and cold-rolled FeNi(Ti) as 1.00328(3), 1.00241(6), and 1.00325(7), respectively. The data of Fig. 3 graphically represent the calculated $c / a$ ratios of all samples made from natural metal plotted as a function of measured iron content. Values from naturally occurring tetrataenite, as reported by 
Albertsen [12] for four different meteorites (Odessa IA, Toluca IA, Dayton IIID, Mincy

Mesosiderite) and confirmed for the NWA 6259 meteorite [44], are included in Fig. 3 for

reference. It can be clearly seen that the axial ratios of all unprocessed samples fall below a value of $c / a \sim 1.0008$ while those of the processed samples fall above a value of $c / a \sim 1.002$. Further, the $c / a$ values determined from data obtained from processed samples are consistent with those of meteoritic L1 ${ }_{0}$ FeNi. The degrees of tetragonality of the processed materials and of the meteoritederived tetrataenite exhibit little variation around the equiatomic composition and then decrease with increased iron content to a value of 1.0020. In contrast, the degree of tetragonality of the unprocessed samples shows little or no trend with iron content.

The variation in the unit cell volumes of all studied FeNi samples as a function of iron content is displayed in Fig. 4, where it can be seen that the unit cell volumes of all synthesized samples, whether of cubic or tetragonal symmetry, are approximately $1 \%$ greater than those of all meteorite-derived tetrataenite ( $\mathrm{L} 1_{0}$ phase) samples. Further, there is very little difference between the unit cell volumes of the cubic unprocessed samples and the tetragonal processed samples, confirming a conservation of unit cell volume upon tetragonal distortion. Additionally, it is noted that the Ti-containing samples consistently exhibit an expanded unit cell lattice relative to the Tifree samples, providing both a relatively larger unit cell volume and a larger $c / a$ ratio.

The tetragonal $c / a$ ratios of deformed and annealed isotopically modified $\mathrm{Fe}^{62} \mathrm{Ni}$ and $\mathrm{Fe}^{62} \mathrm{Ni}(\mathrm{Ti})$ samples were determined as 1.0026(3) and 1.0028(3), respectively. These $c / a$ ratio values are identical (within error) to those of the deformed and annealed samples made from naturally occurring FeNi. It is highly significant that any $\mathrm{L} 1_{0}$ superstructure peaks are absent from all diffraction data, including those obtained from isotopically modified samples. A representative 
neutron diffraction pattern collected from a sample containing ${ }^{62} \mathrm{Ni}$ is provided in Fig. S3. This lack of $\mathrm{L} 1_{0}$ superlattice peaks unequivocally confirms the absence of long-range chemical order in these tetragonal samples. However, a diffuse scattering signal was clearly identified in the neutron diffraction data collected from all isotopically modified samples (Fig. S3), consistent with the presence of local structural correlations in these alloys, in both the deformed pre-annealed and deformed annealed states. The diffuse scattering took the form of a low-intensity, semi-regular waveform concentrated at low $d$-spacings, consistent with that produced by type-1 Krivoglaz defects [45] such as coherent precipitates, and was most clearly observable in the deformed postanneal $\mathrm{Fe}^{62} \mathrm{Ni}$ sample. This diffuse scattering signal is hypothesized to be associated with the formation of a pre-transition state, in accordance with analogous observations of Vlasova et al. of electron diffraction patterns obtained from equiatomic FePd single crystals subjected to annealing and quenching processes [46]. It is preliminarily concluded that the diffuse neutron scattering signal observed from the processed FeNi samples is consistent with local correlations that are related to a very short-range chemical order phenomena.

Initial magnetization curves of the $\mathrm{FeNi}(\mathrm{Ti})$ cold-rolled samples measured both before and after annealing, in sample orientations both parallel and perpendicular to the rolling direction, exhibit an appreciable annealing-induced decrease in the initial susceptibility as well as a $6 \%$ decrease in saturation magnetization, Fig. 5. This decrease in initial susceptibility signals an annealinginduced increase in magnetic anisotropy. Employing the Law of Approach to Ferromagnetic Saturation, the anisotropy constants of the cold-rolled, unannealed state are determined as 12 $\mathrm{kJ} / \mathrm{m}^{3}$ (parallel to the rolling direction) and as $9 \mathrm{~kJ} / \mathrm{m}^{3}$ (perpendicular to the rolling direction). These values, which are two orders of magnitude higher than those reported for quenched fcc $\mathrm{Fe}_{50} \mathrm{Ni}_{50}[40]$, are in agreement with early reports of anisotropy constants of cold-rolled FeNi 
near the equiatomic composition [47]. After annealing, the anisotropy constants of this same sample are found to have increased by approximately $10-12 \%$, to $\sim 1.2 \mathrm{~kJ} / \mathrm{m}^{3}$ for both directions. The origin of this magnetic anisotropy increase may be clarified from results reported by Razee, Staunton and coworkers using ab initio electronic structure calculations to clarify relationships between magnetocrystalline anisotropy, chemical order and lattice distortion in a selection of ferromagnetic compounds $[48,49,50]$. These authors concluded that the magnetocrystalline anisotropy energies may be attributed both to lattice distortion and to chemical ordering, with tetragonalization contributing about $20 \%$ of the and chemical order contributing the remaining $80 \%$ in $\mathrm{L}_{0}$-type CoPt. For comparison, the calculated anisotropy contribution from the measured tetragonal distortion of the FeNi(Ti) sample $(\varepsilon \sim 0.23 \%)$ is 2.4 $\mathrm{kJ} / \mathrm{m}^{3}$. This value is in reasonable agreement with those determined from the Law of Approach to Ferromagnetic Saturation but is only $\sim 0.2 \%$ of the reported value for $\mathrm{L} 1_{0}$-type FeNi [4]. Therefore, the measured anisotropy enhancement of the fully processed samples may be entirely attributed to tetragonal distortion of the structure, and not to the presence of chemical order, in accordance with the neutron diffraction results. Further, the noted $6 \%$ reduction in saturation magnetization upon transition from the A1 to the A6 structure is consistent with the reported 15\% saturation magnetization increase noted upon disordering NWA 6259-derived tetrataenite from the $\mathrm{L} 1_{0}$ to the A1 state [43]. These data facilitate interpretation of Fig. 4: the FeNi unit cell volume is found to experience a significant contraction upon chemically ordering to form the $\mathrm{L}_{0}$ structure. This contraction is associated with a change in the local bonding landscape that fundamentally influences the magnetocrystalline anisotropy and the saturation magnetization of these FeNi phases [43]. 
This current report of a new tetragonal A6-type FeNi phase in the Fe-Ni system is in alignment with a few reports in the literature. Vlasova et al. [46] has recently reported that the A6 structure exists as a symmetry-permitted transitional tetragonal phase in the FePd system, bridging the disordered cubic A1 phase and the chemically ordered tetragonal $\mathrm{L} 1_{0} \mathrm{FePd}$ phase. She predicted that this A6 structure likely exists in other $\mathrm{L} 1_{0}$-forming alloys as well. In fact, Orehotsky et al. $[51,52,53]$ report the existence of a transient phase between the $\mathrm{A} 1$ and $\mathrm{L} 1_{0}$ phases in bulk CoPt samples, with a saturation magnetization that is approximately $5 \%$ lower than that of the A1 phase. Confirmed by X-ray diffraction studies as a non-uniformly strained fcc state [51], it is possible that this transient CoPt phase does in fact possess tetragonal A6-type symmetry.

Finally, the fundamental results reported here provide applied engineering insight into the design of synthesis and processing protocols to develop $\mathrm{L} 1_{0}$-type phase in industrially relevant timescales. It is deduced that the transition to the thermodynamically favored $\mathrm{L}_{0}$ phase from the parent cubic phase in the Fe-Ni system is initially accommodated by a displacive (i.e., diffusionless) transformation from the $\mathrm{A} 1$ to the $\mathrm{A} 6$ phase that may then subsequently proceed to the $\mathrm{L} 1_{0}$ phase via nucleation and growth of nuclei of chemically ordered regions. Processing strategies that offer these capabilities are anticipated to be successful in developing this phase.

\section{Conclusions}

The confirmed tetragonality of the near-equiatomic FeNi-based bulk samples together with the unequivocal absence of long-range chemical ordering establishes that severe plastic deformation followed by appropriate annealing protocols promotes formation of a newly discovered tetragonal phase with the chemically disordered (A6) structure in the FeNi system. The positive identification of the A6-type tetragonal phase in the nominally equiatomic FeNi bulk system produced by metallurgical processing techniques furnishes new knowledge concerning the Fe-Ni 
binary phase diagram as well as indicates positive progress towards achievement of bulk $\mathrm{L}_{0} \mathrm{FeNi}$, with promise for the realization of next-generation advanced permanent magnets.

Acknowledgements: This work was supported in part by the National Science Foundation [grant numbers CMMI-1129433, CMMI-1259736], by the U.S. Office of Naval Research [grant No. N00014-10-1-0553] and by Northeastern University. Student stipend support from Rogers Corporation is gratefully acknowledged. The isotope used in this research was supplied by the United States Department of Energy Office of Science by the Isotope Program in the Office of Nuclear Physics. ADF thanks the UK Science and Technology Facilities Council (STFC), grant number ST/K000934/1 for support. Authors LHL, KB, NCB and AMMA are grateful to the late Distinguished Prof. Emeritus Joseph I Goldstein of the University of Massachusetts and to Dr. Matthew Kramer of the Ames Laboratory, U.S. D.O.E. for helpful discussions. Experiments at the ISIS Pulsed Neutron and Muon Source were supported by a beamtime allocation from the U.K. Science and Technology Facilities Council. Support for the isotope enhancement approach was provided by Dr. Kevin Knight, HRPD, and is gratefully acknowledged. 
Citations

[1] R.F. Tylecote, A History of Metallurgy, 2nd Ed., The Institute of Materials, Brookfield, VT, 1992.

[2] E. Photos, The question of meteoritic versus smelted nickel-rich iron: Archaeological evidence and experimental results, World Archeol. 20 (1989) 403-421.

[3] L. Aitchison, A History of Metals, Volume 1, Macdonald \& Evans, Ltd., London, 1960.

[4] L.H. Lewis, F.E. Pinkerton, N. Bordeaux, A. Mubarok, E. Poirier, J.I. Goldstein, et al., De Magnete et Meteorite : Cosmically Motivated Materials, IEEE Magn. Lett. 5 (2014) 1-4.

[5] K. Smith Stegen, Heavy rare earths, permanent magnets, and renewable energies: An imminent crisis, Energy Policy. 79 (2015) 1-8.

[6] R.W. McCallum, L.H. Lewis, R. Skomski, M.J. Kramer, I.E. Anderson, Practical Aspects of Modern and Future Permanent Magnets, Annu. Rev. Mater. Res. 44 (2014) 451-477.

[7] J.I. Goldstein, J.M. Short, The iron meteorites, their thermal history and parent bodies, Geochim. Cosmochim. Acta. 31 (1967) 1733-1770.

[8] C.W. Yang, D.B. Williams, J.I. Goldstein, A revision of the Fe-Ni phase diagram at low temperatures (<400 C), J. Phase Equilibria. 17 (1996) 522-531.

[9] P. Ehrenfest, Phase changes in the ordinary and extended sense classified according to the corresponding singularities of the thermodynamic potential., Proc. K. Ned. Akad. van Wet. 36 (1933) 153-157.

[10] J.B. Newkirk, A.H. Geisler, D.L. Martin, R. Smoluchowski, Ordering reaction in cobaltplatinum alloys., J. Met. 188 (1950) 1249-1260.

[11] B. Zhang, M. Lelovic, W.A. Soffa, The formation of polytwinned structures in Fe-Pt and Fe- Pd alloys, Scr. Metall. Mater. 25 (1991) 1577-1582.

[12] J.F. Albertsen, Tetragonal lattice of tetrataenite (ordered Fe-Ni, 50-50) from 4 meteorites, Phys. Scr. 23 (1981) 301-306.

[13] K.B. Reuter, D.B. Williams, J.I. Goldstein, Ordering in the Fe-Ni system under electron irradiation, Metall. Trans. A. 20A (1989) 711-718.

[14] T.B. Massalski, H. Okamoto, P.R. Subramanian, L. Kacprzak, eds., Binary Alloy Phase Diagrams, 2nd Ed., ASM International, Materials Park, Ohio, USA., 1990.

[15] O. Dahl, Cold-work deformation and recovery in alloys with orderly atom configuration, Zeitschrift Fuer Met. 28 (1936) 133-138. 
[16] R. Wakelin, E. Yates, A study of the order-disorder transformation in Iron-Nickel alloys in the region FeNi3, Proc. Phys. Soc. Sect. B. 66 (1953) 221-240.

[17] X. Jiang, G. Ice, C.J. Sparks, L. Robertson, P. Zschack, Local atomic order and individual pair displacements of $\mathrm{Fe}_{46.5} \mathrm{Ni}_{53.5}$ and $\mathrm{Fe}_{22.5} \mathrm{Ni}_{77.5}$ from diffuse x-ray scattering studies, Phys. Rev. B. 54 (1996) 3211-3226.

[18] J. Petersen, M. Aydin, J. Knudsen, Mössbauer spectroscopy of an ordered phase (superstructure) of FeNi in an iron meteorite, Phys. Lett. A. 62A (1977) 192-194.

[19] R.S. Clarke, E.R.D. Scott, Tetrataenite--ordered FeNi, a new mineral in meteorites, Am. Mineral. 65 (1980) 624-630.

[20] J. Paulevé, D. D, Orientational superlattice of a Fe-Ni (50-50\%) alloy obtained by neutron irradiation in a magnetic field, Comptes Rendus L'Académie Des Sci. 250 (1960) 3804.

[21] J. Paulevé, D. Dautreppe, J. Laugier, L. Néel, Establishment of an ordered FeNi structure by neutron irradiation, Comptes Rendus L’Académie Des Sci. 254 (1962) 965.

[22] J. Paulevé, D. Dautreppe, J. Laugier, L. Néel, A new order-disorder transition in Fe-Ni (5050), J. Phys. Le Radiux. 23 (1962) 841.

[23] W. Chambron, D. Dautreppe, L. Néel, J. Paulevé, Irradiation aux électrons de $1 \mathrm{MeV}$ d'un alliage Fe-Ni (50-50\%), Comptes Rendus L’Académie Des Sci. 255 (1962) 2037.

[24]. T. Shima, M. Okamura, S. Mitani, K. Takanashi, Structure and magnetic properties for L1 $1_{0^{-}}$ ordered FeNi films prepared by alternate monatomic layer deposition, J. Magn. Magn. Mater. 310 (2007) 2213-2214.

[25] T. Kojima, M. Mizuguchi, K. Takanashi, L1 $1_{0}$-ordered FeNi film grown on Cu-Ni binary buffer layer, J. Phys. Conf. Ser. 266 (2011) 012119.

[26] S. Lee, K. Edalati, H. Iwaoka, Z. Horita, T. Ohtsuki, T. Ohkochi, et al., Formation of FeNi with $\mathrm{L1}_{0}$-ordered structure using high-pressure torsion, Philos. Mag. Lett. 94 (2014) 639-646.

[27] A. Makino, P. Sharma, K. Sato, A. Takeuchi, Y. Zhang, K. Takenaka, Artificially produced rare-earth free cosmic magnet, Sci. Rep. 5 (2015) 16627.

[28] V.F. Sears, Neutron scattering lengths and cross sections, Neutron News. 3 (1992) 26-37.

[29] B. Zhang, L. Lu, M.. Lai, Evolution of vacancy densities in powder particles during mechanical milling, Phys. B Condens. Matter. 325 (2003) 120-129.

[30] K. Detemple, O. Kanert, J. De Hosson, K. Murty, In situ nuclear magnetic resonance investigation of deformation-generated vacancies in aluminum, Phys. Rev. B. 52 (1995) 125-133. 
[31] Kresse T, Li YJ, Boll T, Borchers C, Choi P, Al-Kassab T, Raabe D, Kirchheim

$\mathrm{R}$ : Influence of supersaturated carbon on the diffusion of $\mathrm{Ni}$ in ferrite determined by atom probe tomography, Scripta Materialia 69 (2013) 424 - 427.

[32] Kresse T, Borchers C, Kirchheim R: Vacancy-carbon complexes in bcc-iron: Correlation between carbon content, vacancy concentration, and diffusion coefficient, Scripta Materialia 69 (2013) 690 - 693.

[33] J. Liu, K. Barmak, Interdiffusion in nanometric Fe/Ni multilayer films, J. Vac. Sci. Technol. A Vacuum, Surfaces, Film. 33 (2015) 021510.

[34] H.L. Skriver, Materials Science Databases, Databases CAMP. (2004).

http://guglen.dk/databases/hlsDB/hlsDB.php.>

[35] R.M. Ibberson, Design and performance of the new supermirror guide on HRPD at ISIS, Nucl. Instruments Methods Phys. Res. Sect. A Accel. Spectrometers, Detect. Assoc. Equip. 600 (2009) 47-49.

[36] A.C. Larsen, R.B. Von Dreele, General Structure Analysis System (GSAS). Los Alamos National Laboratory Report LAUR 86-748, Los Alamos, New Mexico, 2000.

[37] B.H. Toby, EXPGUI, a graphical user interface for GSAS, J. Appl. Crystallogr. 34 (2001) 210-213.

[38] A.M. Montes-Arango, N.C. Bordeaux, J. Liu, K. Barmak, L.H. Lewis, L1 10 phase formation in ternary FePdNi alloys, J. Alloys Compd. 648 (2015) 845-852.

[39] S. Chikazumi, Physics of Magnetism, John Wiley and Sons, New York, 1964.

[40] K. Adachi, D. Bonneberg, R. Gersdorf, J.J.M. Franse, K.A. Hempel, K. Kanematsu, et al., Landolt-Börstein: Numerical data and functional relationships in science and technology New Series, Editors in Chief: K. H. Hellwege and O. Madelung. Group III: Crystal and Solid State Physics, Vol. 19: Magnetic Properties of Metals, Subvolume a: 3d, 4d a, Springer-Verlag Berlin Heidelberg, 1986.

[41] R.M. Bozorth, Ferromagnetism, Van Nostrand, New York, 1951.

[42] H.M. Ledbetter, R.P. Reed, Elastic Properties of Metals and Alloys, I. Iron, Nickel, and Iron-Nickel Alloys, J. Phys. Chem. Ref. Data. 2 (1973) 531.

[43] L.H. Lewis, A. Mubarok, E. Poirier, N. Bordeaux, P. Manchanda, A. Kashyap, et al., Inspired by nature: investigating tetrataenite for permanent magnet applications., J. Phys. Condens. Matter. 26 (2014) 064213.

[44] N.C. Bordeaux, Structure-magnetism correlations and chemical order-disorder transformations in ferrous $\mathrm{L}_{1}$-structured compounds, Dissertation, Northeastern University, 2015. 
[45] M.A. Krivoglaz, Theory of diffuse scattering of X-rays and thermal neutrons in solid solutions. III. Account of geometrical distortions of the lattice, Sov. Phys. JETP. 34 (7) (1958) 139.

[46] N.I. Vlasova, a. G. Popov, N.N. Shchegoleva, V.S. Gaviko, L. a. Stashkova, G.S. Kandaurova, et al., Discovery of metastable tetragonal disordered phase upon phase transitions in the equiatomic nanostructured FePd alloy, Acta Mater. 61 (2013) 2560-2570.

[47] G.W. Rathenau, J.L. Snoek, Magnetic anisotropy phenomena in cold-rolled nickel-iron, Phys. VIII. 6 (1941) 555.

[48] S.S.A. Razee, J.B. Staunton, B. Ginatempo, E. Bruno, F.J. Pinski, Ab initio theoretical description of the dependence of magnetocrystalline anisotropy on both compositional order and lattice distortion in transition metal alloys, Phys. Rev. B. 64 (2001) 014411.

[49] S.S.A. Razee, J.B. Staunton, B. Ginatempo, F.J. Pinski, E. Bruno, Ab initio theoretical description of the interrelation between magnetocrystalline anisotropy and atomic short-range order, Phys. Rev. Lett. 82 (1999) 5369.

[50] J.B. Staunton, S. Ostanin, S.S.A. Razee, B. Gyorffy, L. Szunyogh, B. Ginatempo, et al., Long-range chemical order effects upon the magnetic anisotropy of $\mathrm{FePt}$ alloys from an ab initio electronic structure theory, J. Phys. Condens. Matter. 16 (2004) S5623.

[51] J. Orehotsky, J.L. Orehotsky, Kinetics of the order-disorder transformation in Pt1Co1, J. Appl. Phys. 61 (1987) 1210.

[52] J. Orehotsky, J.L. Orehotsky, An anomalous magnetic effect in the Pt1Co1 order-disorder alloy system, J. Appl. Phys. 61 (1987) 4240.

[53] J. Orehotsky, Magnetic and crystallographic characteristics of the order-disorder phase transformation in Pt1Co1, IEEE Trans. Magn. 25 (1989) 3791-3793. 
a)

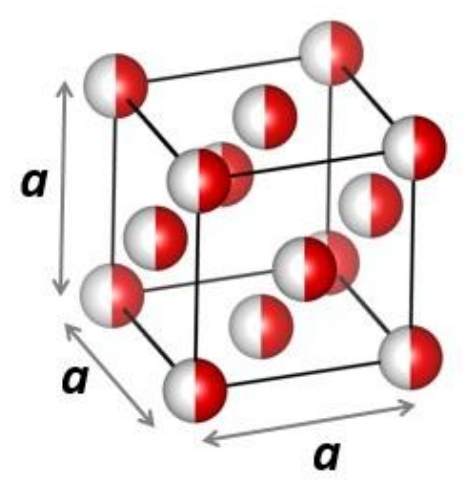

b)

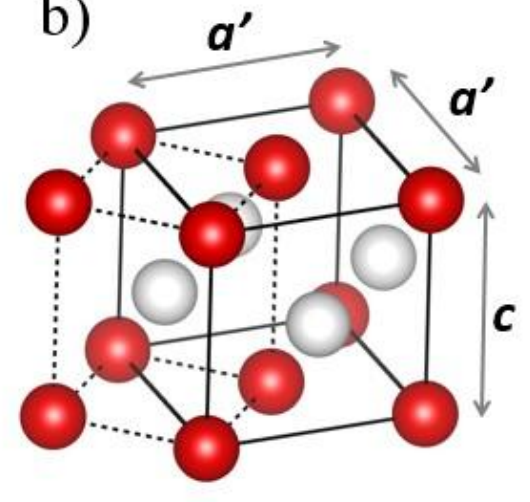

Fig. 1. Representations of the cubic (Al) unit cell and the tetragonal ( $\left.L 1_{0}\right)$ unit cell. The unit cells of a) the disordered face-centered cubic (fcc) and b) the tetragonal chemically ordered $\mathrm{L} 1_{0}$ crystal structures. The lattice parameters are identified, and the half-shaded spheres of depiction a) indicate an equal probability of lattice site occupancy for either Fe or Ni. For clarity, depiction b) shows the same orientation of the basis vectors of the cubic unit cell and a non-standard, C-centered tetragonal unit cell to indicate the $\mathrm{L} 1_{0}$ structure, with the primitive unit cell delineated by the dashed black lines. 

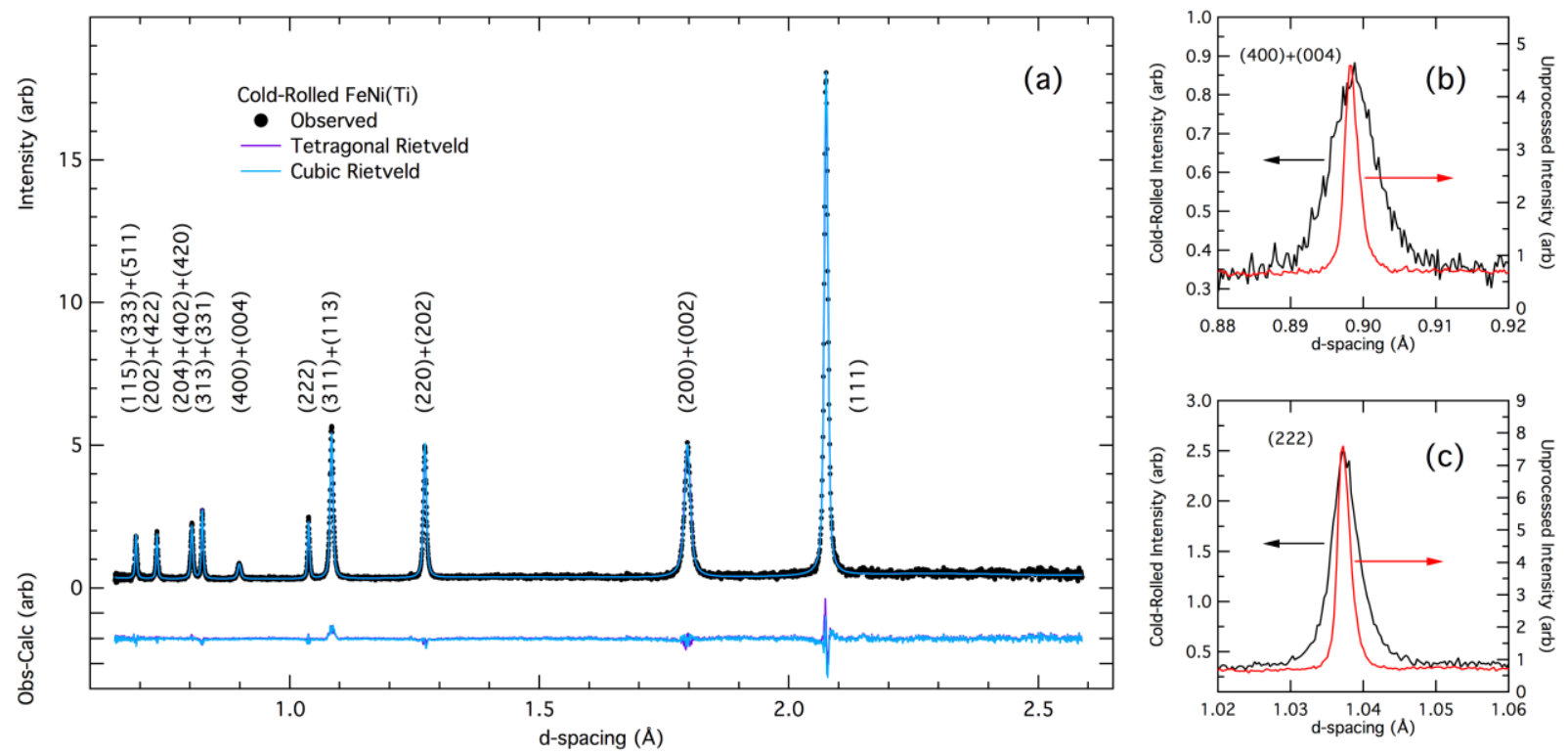

Fig. 2. Neutron diffraction pattern obtained for the cold-rolled annealed FeNi(Ti) sample. (a) Observed data points are displayed with the calculated tetragonal (purple)_and cubic (cyan) Rietveld refinement fits overlaid. The Miller indices $\left(\mathrm{hkl}\right.$ ) of the Bragg peaks (for a $\mathrm{C} 4 / \mathrm{mmm}$ representation of the $\mathrm{L} 1_{0}$ unit cell) are included for reference. Differences between the observed data and the calculated patterns are displayed in the traces located below the peaks. (b) Detailed view of the (400) fundamental Bragg peak data derived from cold-rolled annealed $\mathrm{FeNi}(\mathrm{Ti})$ in both the unprocessed (red trace) and processed (black trace) states that illustrate processing-induced broadening of this peak, consistent with symmetry lowering. (c) Detailed view of the (222) fundamental Bragg peak derived from cold-rolled annealed $\mathrm{FeNi}(\mathrm{Ti})$ in both the unprocessed (red trace) and processed (black trace) states that emphasize processinginduced broadening of this peak. 


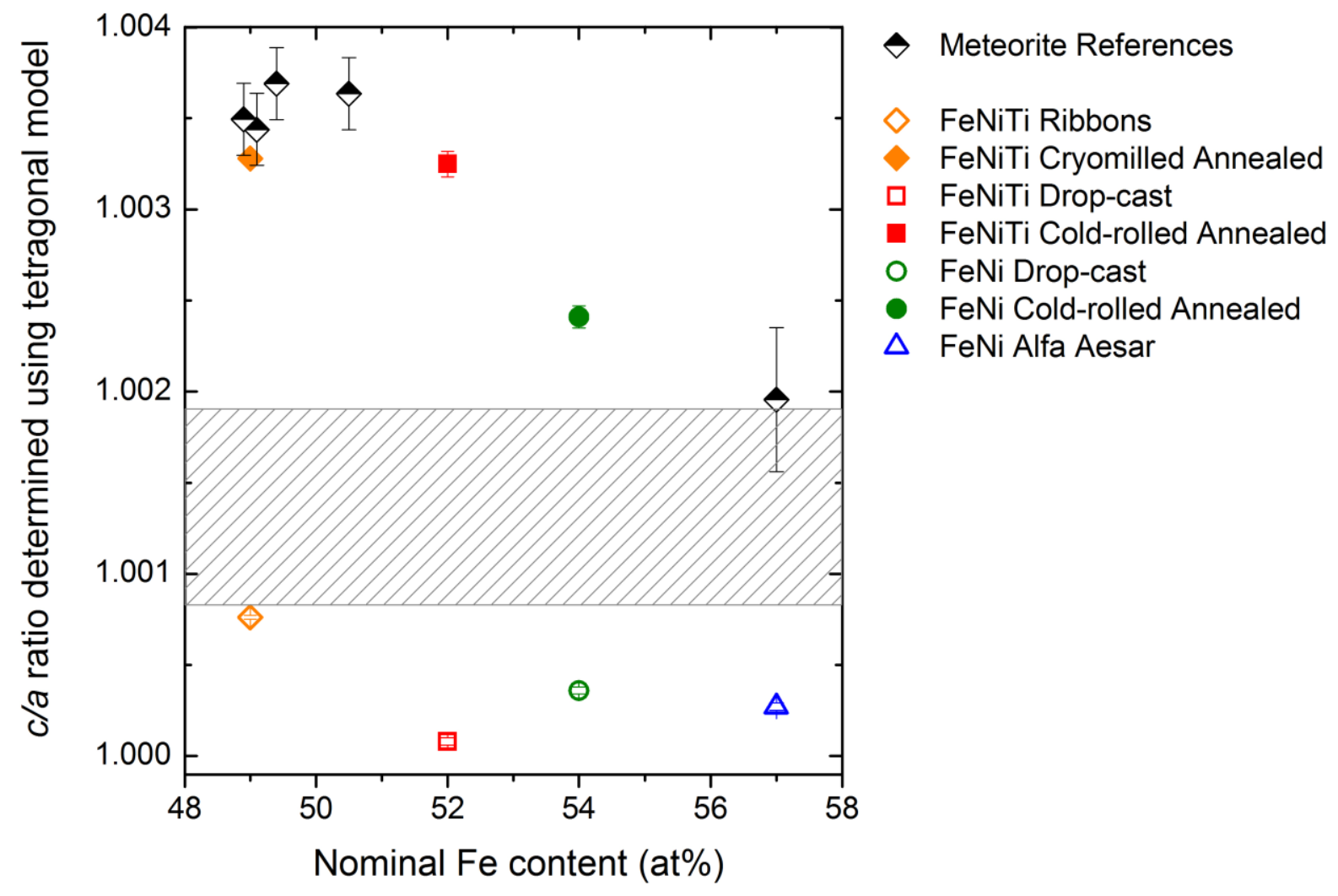

Fig. 3. Sample tetragonality as a function of iron content for studied FeNi-based samples. Graphical depiction of calculated $c / a$ ratios as determined from the tetragonal Rietveld model applied to neutron data derived from both unprocessed and processed FeNi-based samples made from natural metal sources. Published lattice parameter data from natural tetrataenite obtained from meteorites are included. Error bars represent the propagated lattice parameter standard deviations derived via Rietveld refinement with $\sim 7800$ data points diffraction pattern. The shaded region indicates a band of values that separates $c / a$ ratio determinations from unprocessed samples and those determined from processed samples; the displayed $c / a$ ratio values reported from meteorite-derived tetrataenite corroborates the conclusion that all unprocessed FeNi-based samples studied adopt the cubic structure while all processed FeNi-based samples studied adopt the tetragonal structure. 


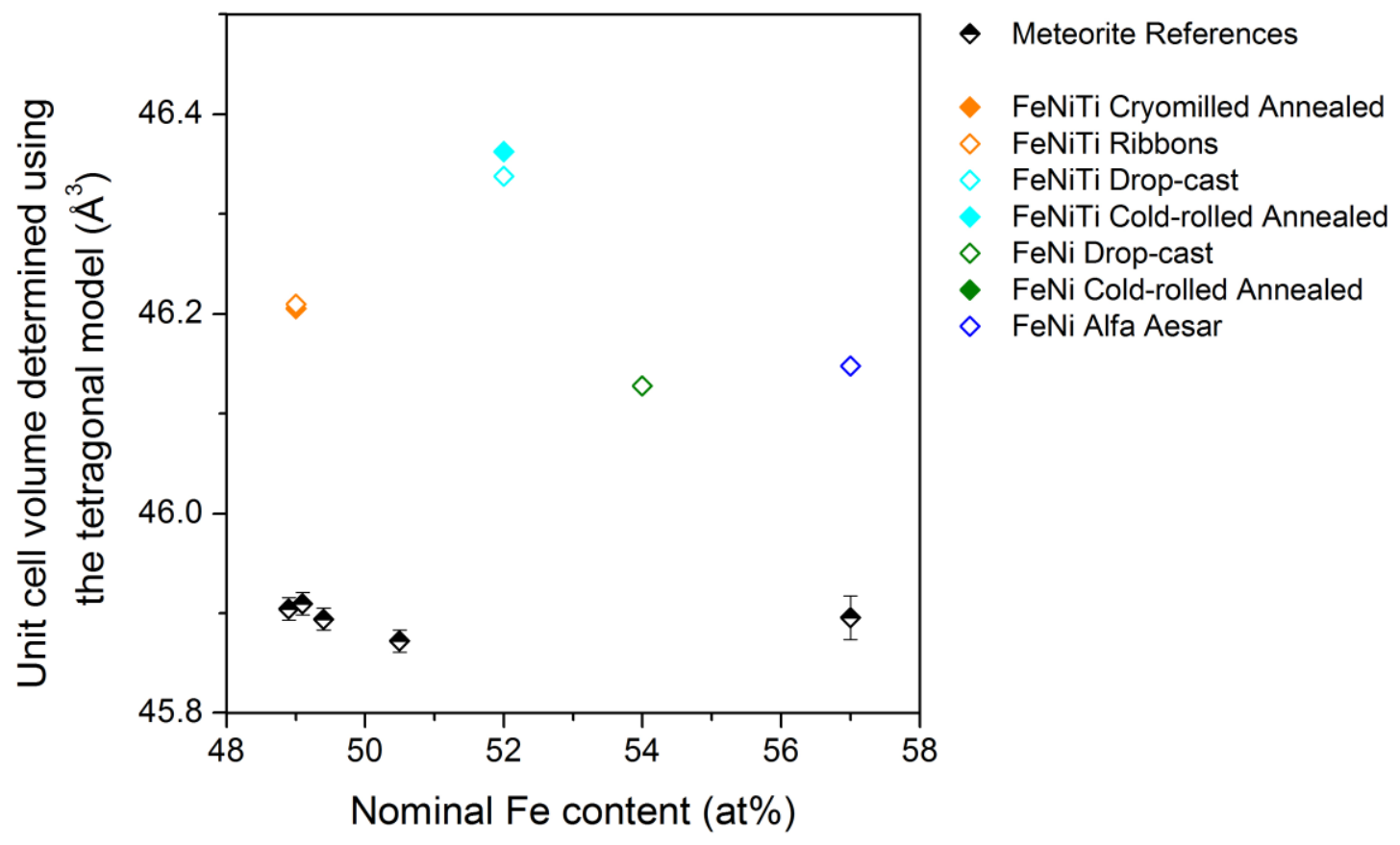

Fig. 4. Trends of unit cell volume as a function of Fe content in FeNi-based samples and meteorites. A graphical depiction is provided of unit cell volumes determined from the tetragonal Rietveld model applied to neutron data collected from unprocessed and processed FeNi-based samples made from natural metal sources. Error bars represent the propagated lattice parameter standard deviations derived via Rietveld refinement with $\sim 7800$ data points diffraction pattern. Published data from natural meteoritederived tetrataenite are included. The unit cell volumes of all synthesized samples are larger than those of all meteoritic tetrataenite by approximately $1 \%$. 


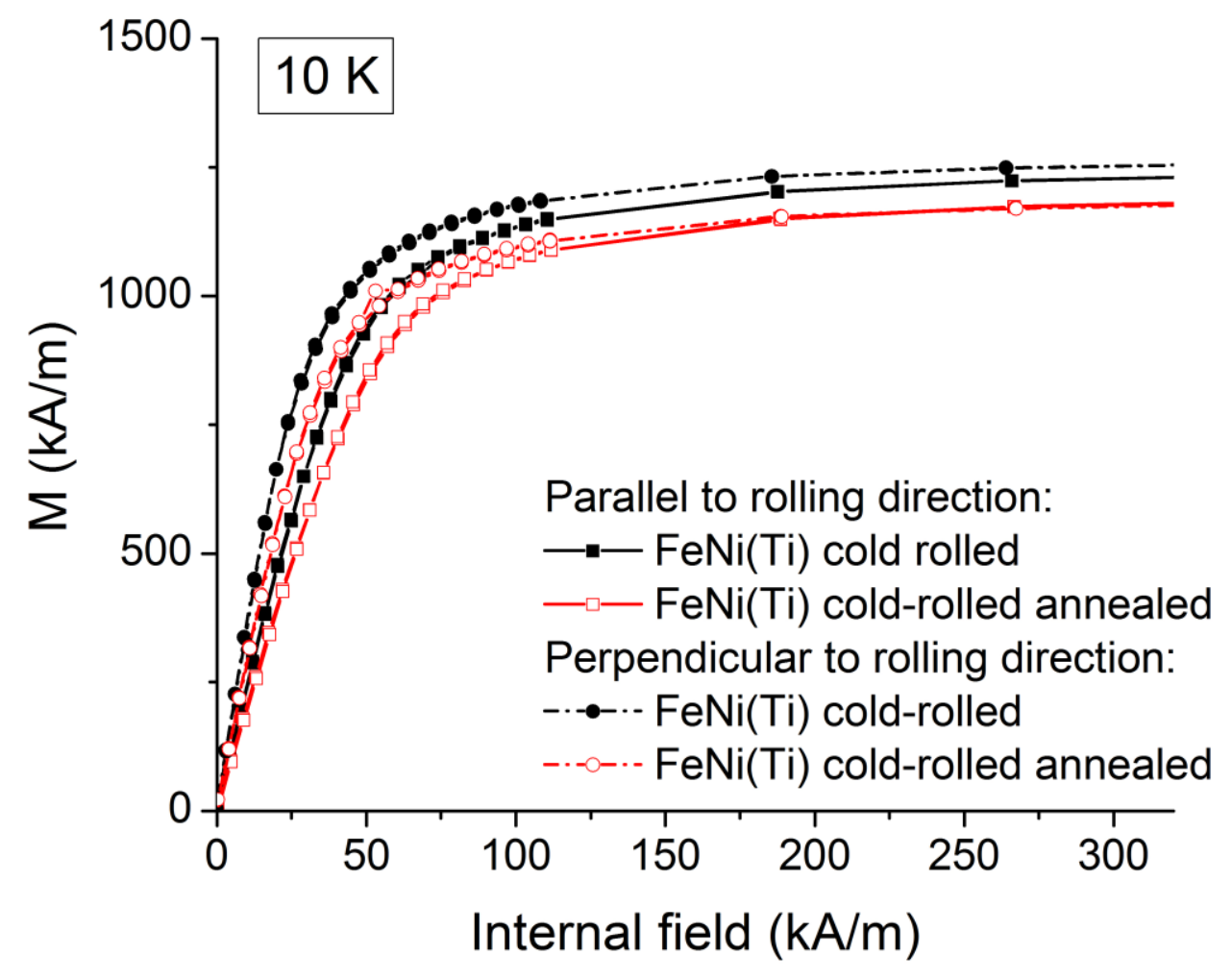

Fig. 5. Initial magnetization data obtained at $T=10 \mathrm{~K}$ for the cold-rolled FeNi(Ti) sample measured before and after annealing. Data were collected in the in-plane directions both parallel and perpendicular to the rolling direction to reveal a decrease in initial susceptibility and saturation magnetization achieved upon annealing. 
Table 1. Descriptions of compositions, types and processing of studied samples.

\begin{tabular}{lll}
\hline Assessed composition & Element type and processing method & Designation \\
\hline $\mathrm{Fe}_{54} \mathrm{Ni}_{46}$ & Natural abundance elements, cold-rolled & $\mathrm{FeNi}$, cold-rolled \\
$\mathrm{Fe}_{52} \mathrm{Ni}_{46} \mathrm{Ti}_{2}$ & Natural abundance elements, cold-rolled & $\mathrm{FeNi}(\mathrm{Ti})$, cold-rolled \\
$\mathrm{Fe}_{49} \mathrm{Ni}_{50} \mathrm{Ti}_{1}$ & Natural abundance elements, cryomilled & $\mathrm{FeNi}(\mathrm{Ti})$, cryomilled \\
$\mathrm{Fe}_{51}\left({ }^{62} \mathrm{Ni}_{49}\right)$ & $\begin{array}{l}\text { Isotopic Ni, natural abundance } \mathrm{Fe} \text {, cold- } \\
\text { rolled }\end{array}$ & $\mathrm{Fe}^{62} \mathrm{Ni}$, cold-rolled \\
$\mathrm{Fe}_{50}\left({ }^{62} \mathrm{Ni}_{48}\right) \mathrm{Ti}_{2}$ & Isotopic Ni, natural abundance Fe and $\mathrm{Ti}$, & $\mathrm{Fe}^{62} \mathrm{Ni}(\mathrm{Ti})$, cold-rolled \\
& cold-rolled & \\
$\mathrm{Fe}_{57} \mathrm{Ni}_{43}$ powder & Commercial Alfa Aesar powder, -325 mesh, 99.5+\% purity \\
$\mathrm{Fe}_{57} \mathrm{Ni}_{43}$ from NWA 6259 & Meteoritic origin & \\
$\mathrm{meteorite}$ & & \\
\hline
\end{tabular}


Fe
Ni

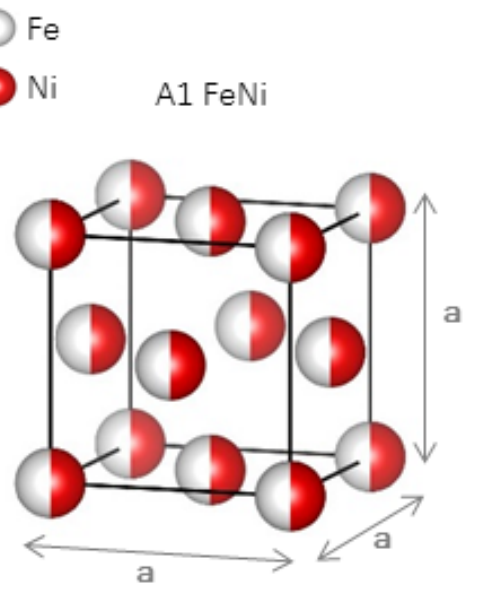

A1 FeNi
A6 FeNi:

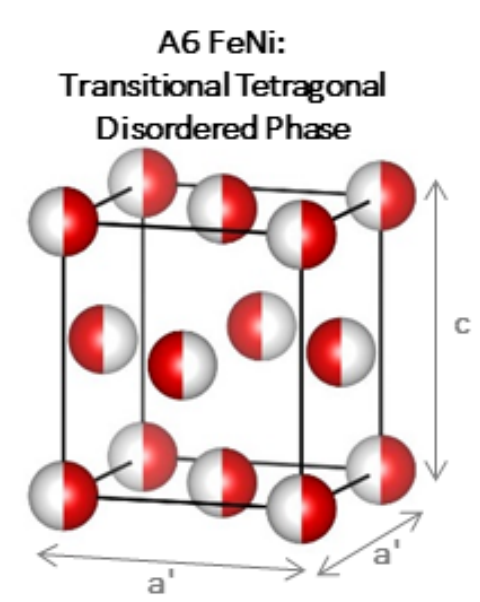

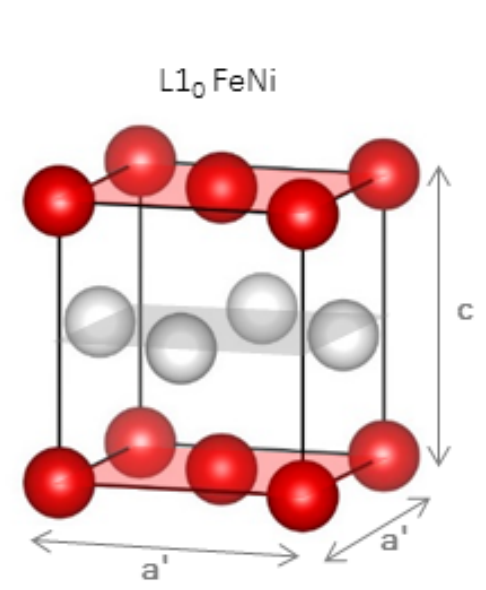

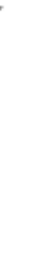

